\title{
ON BERNSTEIN ALGEBRAS WHICH ARE TRAIN ALGEBRAS
}

\author{
by SEBASTIAN WALCHER
}

(Received 9th May 1990)

\begin{abstract}
The class of non-associative algebras over a field of characteristic zero named in the title is studied using a result of Ouattara [9]. As an application, the differential equation for overlapping generations in the timecontinuous model is solved.
\end{abstract}

1980 Mathematics subject classification (1985 Revision): 17D99.

\section{Introduction}

A commutative non-associative algebra $A$ of finite dimension over a field $K$ is called a Bernstein algebra if there is a nontrivial homomorphism $\omega: A \rightarrow K$ and if the identity

$$
x^{2} x^{2}-\omega(x)^{2} x^{2}=0
$$

holds in $A$.

This class of algebras was introduced by Holgate [4], following the original work of Bernstein [2] and subsequent investigations by Lyubich [5] on idempotent quadratic maps from a real simplex into itself. A summary of known results on Bernstein algebras (up to 1980) is given in Wörz-Busekros [8], which will also be used as a basic reference on algebras in genetics. All definitions not explicitly stated here can be found in this monograph.

Bernstein algebras are not necessarily genetic algebras in the sense of Schafer [6]; indeed there are Bernstein algebras which are not even train algebras, cf. Wörz-Busekros [8].

In this note Bernstein algebras which are train algebras are considered. $K$ is assumed to be of characteristic zero throughout, although this hypothesis could be weakened for some of the results.

As follows from a result of Outtara [9], the rank polynomial of a Bernstein train algebra is uniquely determined by its degree. This result admits an interpretation as a "stationarity principle" which is different from (1), and this in turn enables us to solve the differential equation for overlapping generations in the time-continuous model (for $K=\mathbb{R})$ in closed form. In particular, the long-term behaviour of the solutions can easily be determined.

I thank the referee for valuable comments and suggestions. 


\section{The rank equation}

For every $x \in A$ we define $L(x) \in \operatorname{Hom}(A, A)$ by $L(x) y:=x y$. Powers of an element $x$ are defined by $x^{k}:=L(x)^{k-1} x$ for all $k \in \mathbb{N}$.

Linearizing (1), one obtains

$$
x^{2}(x y)=\frac{1}{2} \omega(x)^{2} x y+\frac{1}{2} \omega(x y) x^{2}
$$

and in particular

$$
x^{2} x^{i}=\frac{1}{2} \omega(x)^{2} x^{i}+\frac{1}{2} \omega(x)^{i} x^{2}
$$

for all $x \in A$ and all $i \geqq 2$.

We will frequently use differentiation ( $=$ linearization) of polynomial maps as a tool. Since $K$ is infinite, this causes no problem. The "product rule" for differentiation of maps stemming from multilinear maps will be used without further mentioning.

For $k \geqq 1$ let $p_{k}(x):=x^{k}$. For $k \geqq 2$ we have

$$
D p_{k}(x) y=2 L(x)^{k-1} y+\sum_{j=0}^{k-3} L(x)^{j}\left(x^{k-j-1} \cdot y\right)
$$

in every commutative algebra; in particular

$$
D p_{k}(x) x^{2}=2 x^{k+1}+\sum_{j=0}^{k-3} L(x)^{j}\left(x^{k-j-1} \cdot x^{2}\right)
$$

For Bernstein algebras, we obtain from (2)

$$
D p_{k}(x) x^{2}=2 x^{k+1}+\frac{1}{2} \sum_{j=0}^{k-3} L(x)^{j}\left(\omega(x)^{k-j-1} x^{2}\right)+\frac{1}{2} \sum_{j=0}^{k-3} L(x)^{j}\left(\omega(x)^{2} x^{k-j-1}\right)
$$

and finally

$$
D p_{k}(x) x^{2}=2 x^{k+1}+\frac{1}{2} \sum_{j=0}^{k-3} \omega(x)^{k-j-1} x^{j+2}+\frac{1}{2}(k-2) \omega(x)^{2} x^{k-1}
$$

For any polynomial $\tilde{g}(\tau)=\sum_{i=0}^{n} \beta_{i} \tau^{i}$ in one indeterminate, we define $g: A \rightarrow A$ by $g(x)=\sum_{i=0}^{n} \beta_{i} \omega(x)^{n-i} x^{i}$. In particular, for $r \geqq 3$ let

$$
f_{r}(\tau):=\tau^{2}(\tau-1)\left(\tau-\frac{1}{2}\right)^{r-3}
$$

Theorem 2.1 (Ouattara [9]). Let $A$ be a Bernstein train algebra, and the degree of its rank equation equal to $r \geqq 3$. Then the rank equation is given by $f_{r}(x)=0$. 
This is actually not quite the theorem stated by Outtara [9, Thm. 2.5], which starts from a nilpotency condition on certain elements of $N:=\operatorname{Ker} \omega$. We will show that in a Bernstein train algebra $\left.L(x)\right|_{N}$ is nilpotent for every $x \in N$, thus Ouattara's additional condition always holds. We abbreviate $M(x):=\left.L(x)\right|_{N}$ for $x \in A$.

Since $x^{r}=0$ for all $x \in N$, we obtain by differentiation

$$
2 M(x)^{r-1}+\sum_{j=0}^{r-3} M(x)^{j} M\left(x^{r-j-1}\right)=0
$$

for all $x \in N$.

On the other hand, $M\left(x^{2}\right) M(x)=0$ for all $x \in N$ follows from the linearization of (1), and we will show that

$$
M\left(x^{i}\right) M(x)^{i-1}=0
$$

holds for all $i \geqq 2$ by induction.

Indeed, starting from (5), differentiating and applying to $x^{2}$ we obtain, using $D p_{i}(x) x^{2}=2 p_{i+1}(x)$ for $x \in N$,

$$
2 M\left(x^{i+1}\right) M(x)^{i-1}+M\left(x^{i}\right) \sum_{j=0}^{i-2} M(x)^{j} M\left(x^{2}\right) M(x)^{i-2-j}=0
$$

and

$$
2 M\left(x^{i+1}\right) M(x)^{i-1}+M\left(x^{i}\right) M(x)^{i-2} M\left(x^{2}\right)=0
$$

from $M\left(x^{2}\right) M(x)=0$. Multiplying by $M(x)$ from the right completes the induction.

Thus, multiplying (4) by $M(x)^{r-2}$ from the right shows $M(x)^{2 r-3}=0$.

We note that the only possible rank equation of degree 2 is $x^{2}-\omega(x) x=0$, thus the picture is complete.

The polynomial maps $f_{l}(l \geqq 3)$ have an interesting property in arbitrary Bernstein algebras. First we note

$$
f_{l+1}(x)=x \cdot f_{l}(x)-\frac{1}{2} \omega(x) f_{l}(x) \text { for all } l \geqq 3 .
$$

Lemma 2.2. $D f_{l}(x) x^{2}=2 x \cdot f_{l}(x)$ for all $l \geqq 3$.

Proof. For $l=3$, this is elementary, and we proceed by induction. Let $f_{l}(\tau)=$ $\sum_{i=2}^{l} \gamma_{, l} \tau^{i}$ and note $\sum_{i=2}^{l} \gamma_{i, l}=\widetilde{f}_{l}(1)=0$.

$$
\begin{aligned}
D f_{l+1}(x) x^{2} & =x^{2} \cdot f_{l}(x)-\frac{1}{2} \omega(x)^{2} f_{l}(x)+x \cdot\left(D f_{l}(x) x^{2}\right)-\frac{1}{2} \omega(x)\left(D f_{l}(x) x^{2}\right) \\
& =x^{2} \cdot f_{l}(x)-\frac{1}{2} \omega(x)^{2} f_{l}(x)+2 x \cdot\left(x \cdot f_{l}(x)\right)-\omega(x)\left(x \cdot f_{l}(x)\right),
\end{aligned}
$$


where the induction hypothesis has been used.

The third and fourth terms on the right hand side combine to $2 x \cdot f_{l+1}(x)$, using (6), the first term, by virtue of (2), equals

$$
\frac{1}{2} \omega(x)^{2} f_{l}(x)+\frac{1}{2}\left(\sum_{i=2}^{l} \gamma_{i, l} \omega(x)^{l-i} \omega(x)^{i}\right) x^{2}=\frac{1}{2} \omega(x)^{2} f_{l}(x)
$$

Thus the assertion is proved.

Now define recursively

$$
g_{0}(x):=x, g_{k+1}(x):=\frac{1}{k+1} \sum_{j=0}^{k} g_{j}(x) g_{k-j}(x) .
$$

In particular, $g_{1}(x)=x^{2}$ and $g_{2}(x)=x^{3}$. Note that $g_{k}$ is homogeneous of degree $k+1$. Easy induction shows

$$
D g_{k}(x) x^{2}=(k+1) g_{k+1}(x)
$$

for all $x \in A$ and all $k \geqq 0$. (This is true for any commutative algebra.)

Lemma 2.3. For every Bernstein algebra and every $l \geqq 2$ the identity $g_{l}(x)-$ $\omega(x) g_{l-1}(x)=\left(2^{l-1} / l !\right) f_{l+1}(x)$ is satisfied.

Proof. For $l=2$ we have

$$
g_{2}(x)-\omega(x) g_{1}(x)=x^{3}-\omega(x) x^{2}=f_{3}(x) .
$$

We proceed by induction. Differentiate

$$
g_{l}(x)-\omega(x) g_{l-1}(x)=\frac{2^{l-1}}{l !} f_{l+1}(x)
$$

and apply to $x^{2}$. The left hand side yields, observing (8),

$$
\begin{aligned}
& D g_{l}(x) x^{2}-\omega(x) D g_{l-1}(x) x^{2}-\omega(x)^{2} g_{l-1}(x) \\
& \quad=(l+1) g_{l+1}(x)-l \omega(x) g_{l}(x)-\omega(x)^{2} g_{l-1}(x) \\
& \quad=(l+1)\left(g_{l+1}(x)-\omega(x) g_{l}(x)\right)+\omega(x)\left(g_{l}(x)-\omega(x) g_{l-1}(x)\right) \\
& \quad=(l+1)\left(g_{l+1}(x)-\omega(x) g_{l}(x)\right)+\omega(x) \cdot \frac{2^{l-1}}{l !} f_{l+1}(x)
\end{aligned}
$$


by induction hypothesis.

Using Lemma 2.2 and (6) we obtain on the right hand side

$$
\frac{2^{l-1}}{l !} D f_{l+1}(x) x^{2}=\frac{2^{l-1}}{l !} \cdot 2 x \cdot f_{l+1}(x)=\frac{2^{l-1}}{l !}\left(2 f_{l+2}(x)+\omega(x) f_{l+1}(x)\right) \text {. }
$$

Comparing both sides completes the induction.

Since $f_{r}(x)=0$ implies $f_{l}(x)=0$ for all $l>r$, we have:

Theorem 2.4. If the Bernstein algebra $A$ satisfies a train equation of minimal degree $r \geqq 3$, then $g_{k}(x)-\omega(x) g_{k-1}(x)=0$ for all $x \in A$ and all $k \geqq r-1$.

Remark. Trivially, this is also true for $r=2$. Obviously a Bernstein algebra satisfies a train equation if and only if the identity $g_{l}(x)-\omega(x) g_{l-1}(x)=0$ holds for some $l$.

In applications interest is restricted to elements of the hyperplane $H=\{x \in A: \omega(x)=$ 1\}. Theorem 2.4 shows that in a Bernstein train algebra $g_{r-1}(x)=g_{r}(x)=\cdots$ for all $x \in H$. Thus one has another "stationarity principle" for these algebras. An application will be discussed in the following section.

\section{Application to continuously overlapping generations}

In this section we assume $K=\mathbb{R}$. If heredity in a population is described by squaring in the algebra $A$ and if generations overlap continuously, then, according to Andreoli [1] and Heuch [3] the behaviour of the population is governed by the Riccati differential equation

$$
\dot{y}=y^{2}-y
$$

on the (invariant) hyperplane $H$. For Schafer genetic algebras Heuch [3] showed that this equation can be solved with elementary functions (although his explicit formula needs a few corrections). For Bernstein algebras the solution of (9) seems to be unknown. In the following we find the explicit solution for a Bernstein train algebra. This solution is interesting even for genetic algebras because the longterm behaviour of solutions cannot directly be found from Heuch's results. (In general, there are train roots equal to $\frac{1}{2}$.)

We first consider the homogeneous equation

$$
\dot{y}=y^{2}
$$

in the algebra $A$. Some of the results cited in the following are taken from [7]; they can, however, also easily be verified by direct inspection. 
Denote by $G(x, t)$ the solution of (10) with initial value $x$ at $t=0$.

Lemma 3.1. Let $A$ be a Bernstein algebra. Then

(i) $G(x, t)=\sum_{k=0}^{\infty} g_{k}(x) t^{k}$

(ii) If $f_{r+1}(x)=0$ for all $x \in A$, then

$$
G(x, t)=\sum_{k=0}^{r-2} g_{k}(x) t^{k}+\frac{t^{r-1}}{1-t \omega(x)} g_{r-1}(x)
$$

Proof. (i) is correct for any commutative algebra. Note that the existence of a power series expansion for $G(x, t)$ is guaranteed by the Cauchy-Kovalevsky theorem. The assertion then follows by substituting this series into (10); cf. [7].

(ii) By Lemma 2.3 and Theorem 2.4 we have $g_{r+l}(x)=\omega(x)^{l+1} g_{r-1}(x)$ for all $l \geqq 0$. Hence

$$
G(x, t)=\sum_{k=0}^{r-2} g_{k}(x) t^{k}+t^{r-1} g_{r-1}(x) \cdot\left(\sum_{j=0}^{\infty} t^{j} \omega(x)^{j}\right)
$$

the assertion follows.

It is known that the solution of (9) can be found from the solution of (10); cf. [7].

Let $y(t)$ be a solution of (9) with $y(0)=x$. Define $z(t)$ by $y(t)=e^{-t} z(t)$. Substituting into (9) yields $-e^{-t} z(t)+e^{-t} \dot{z}(t)=e^{-2 t} z(t)^{2}-e^{-t} z(t)$, hence $z(t)$ satisfies the non-autonomous equation

$$
\dot{z}=e^{-t} z^{2}
$$

and $z(0)=x$.

It is immediately verified that $z(t)=G\left(x, 1-e^{-t}\right)$ is the solution of the given initial value problem and we have $y(t)=e^{-t} G\left(x, 1-e^{-t}\right)$. Restriction to $H$ and combination of the results shows:

Theorem 3.2. Let the Bernstein algebra A satisfy $f_{r+1}(x)=0$. Then the solution of (9) for the initial value $x \in H$ is

$$
y(t)=e^{-t}\left(\sum_{k=0}^{r-2} g_{k}(x)\left(1-e^{-t}\right)^{k}\right)+\left(1-e^{-t}\right)^{r-1} g_{r-1}(x)
$$

Corollary 3.3. For $t \rightarrow \infty, y(t) \rightarrow g_{r-1}(x)$.

It follows from general theory that $g_{r-1}(x)$ is a stationary solution of (9), i.e. an 
idempotent of $A$. This can also be found directly (for arbitrary $K$ ) from the recursion (7).

Finally, let us indicate that the results of Section 2 also allow to find the solution of (9) for an arbitrary Bernstein algebra in a (somewhat) closed form: From Lemma 2.3 one has by induction for $x \in H \quad g_{l}(x)=g_{1}(x)+f_{3}(x)+\cdots+\left(2^{l-1} / l !\right) f_{l+1}(x)$ for all $l \geqq 2$; furthermore $f_{l+1}(x)=S(x) f_{l}(x)$ with $S(x)=L(x)-\frac{1}{2} I d$ for all $l \geqq 3$, according to (6) and hence

$$
g_{l}(x)=g_{1}(x)+\left(\sum_{j=0}^{l-2} \frac{2^{j+1}}{(j+2) !} S(x)^{j}\right) f_{3}(x)
$$

This shows for the solution of $(10)$ :

$$
G(x, t)=g_{0}(x)+\frac{t}{1-t} g_{1}(x)+\frac{t^{2}}{1-t}\left(\sum_{j=0}^{\infty} \frac{2^{j+1}}{(j+2) !} t^{j} S(x)^{j}\right) f_{3}(x)
$$

when $x \in H$. The solution of (9) follows as before.

For given $x$, determination of the solution involves evaluation of the series for $S(x)$. This can be done, at least in principle: Note

$$
\begin{aligned}
\sum_{j=0}^{\infty} \frac{2^{j+1}}{(j+2) !} z^{j} & =2 \sum_{j=0}^{\infty} \frac{(2 z)^{j}}{(j+2) !}=\frac{2}{(2 z)^{2}}(\exp (2 z)-2 z-1) \\
& =: \gamma(z) \text { for all } z \in \mathbb{C} .
\end{aligned}
$$

Then $\gamma(B)$ can be found for any $B \in \operatorname{Hom}(A, A)$, provided the Jordan canonical form of $B$ is known. In this sense, the solution of a given initial value problem is reduced to linear algebra. Of course, it is hard to obtain global information from the given formula; hence results about special cases are still valuable.

\section{REFERENCES}

1. G. ANDreoli, Algebre non associative e sistemi differenziati di Riccati in un problema di Genetica, Ann. Mat. Pura Appl. 49 (1960), 97-116.

2. S. Bernstein, Solution of a mathematical problem connected with the theory of heredity, Ann. Math. Statist. 13 (1942), 53-61.

3. I. Heuch, Genetic algebras and time continuous models. Theor. Pop. Bio. 4 (1973), 133-144.

4. P. Holgate, Genetic algebras satisfying Bernstein's stationarity principle, J. London Math. Soc. (2) 9 (1975), 613-623.

5. Yu. I. Lyubich, Basic concepts and theorems of evolution genetics of free populations, Russian Math. Surveys 26 (1971), 51-123.

6. R.D. Schafer, Structure of genetic algebras, Amer. J. Math. 71 (1949), 121-135.

7. S. WALCher, Algebras and differential equations (Hadronic Press, to appear). 
8. A. Wórz-Busekros, Algebras in genetics (Springer Lecture Notes in Biomathematics 36, (1980)).

9. M. Ouatrara, Sur les algèbres de Bernstein qui sont des T-algèbres, Lin. Alg. Appl. 148 (1991), 171-178.

Mathematisches Insttitut der TU München

PostFach 202420

D-8000 MÜNCHEN 2

GeRMANY 